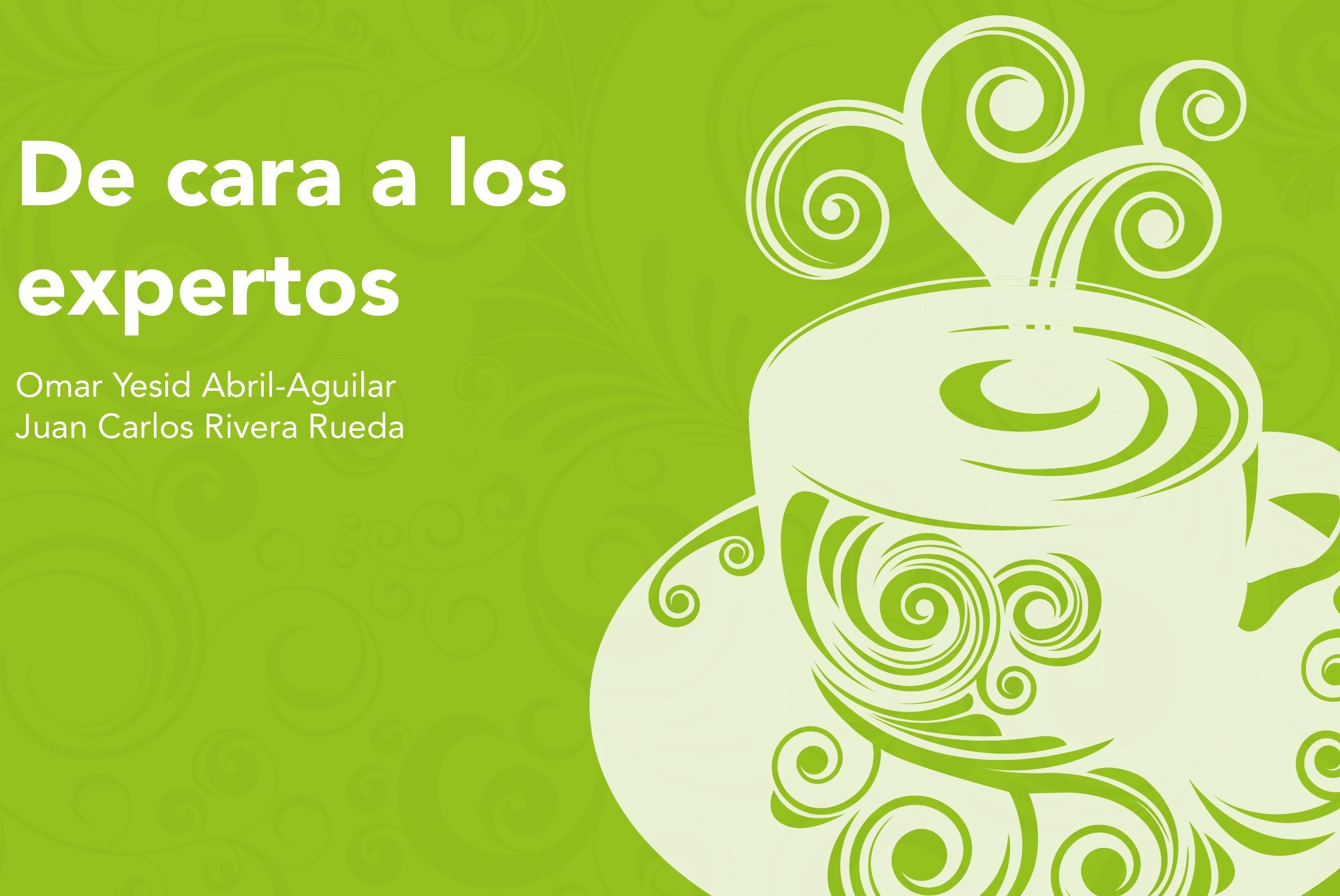




\title{
Modelos educativos, pedagogía y didáctica en educación virtual
}

\author{
: Omar Yesid Abril-Aguilar \\ Universidad Ean \\ oabrilag6521@universidadean.edu.co \\ Juan Carlos Rivera Rueda \\ Universidad Ean \\ jcrivera@universidadean.edu.co \\ DOI: https://doi.org/10.21158/2357514x.v7.n2.2019.2529
}

(c) $(\mathrm{i})\left(\mathrm{BY}_{\mathrm{BC}}\right.$

\section{RESUMEN}

La evolución en la educación universitaria se ha dado gracias al apoyo de las nuevas tendencias tecnológicas y al constante perfeccionamiento de los accesos a plataformas educativas, como, por ejemplo, Blackboard®, Moodle ${ }^{\circledR}$ o Canvas, que han permitido a la educación a distancia, virtual o semipresencial explorar un nuevo horizonte —en campos tales como la pedagogía y la didáctica educativa- encaminada a fomentar nuevas estrategias y posibilidades de conocimiento, lo cual en generaciones anteriores era impensable dado que la única alternativa que imperaba exigía estar presente en un espacio físico bajo un horario establecido. En la última década todo ha cambiado de manera exponencial y dichos requisitos se encuentran en un lugar accesible a todos denominado «la nube» o cloud en inglés. En esta, la educación se expresa y se entiende de un modo distinto con el fin de cambiar la forma de aprender del estudiante y desarrollar un nuevo pensamiento acorde con esta modalidad de estudio que trae consigo una inherente necesidad de autodisciplina y autoaprendizaje, facultades que fungen como pilares de los conceptos de trabajo autónomo para la consecución de una formación profesional integral.

Palabras clave: profesional; plataformas; educación a distancia; virtual; didáctica; nube; estrategias; pensamiento 


\section{Introducción}

La educación moderna, entendida como la transformación radical de los elementos que componen la formación presencial y la relación docente-alumno, ha logrado apoyarse en los avances tecnológicos para generar nuevas oportunidades de conocimiento y abrir la puerta a nuevos horizontes frente la interdisciplinariedad del saber proveniente y convergente entre varias culturas.

La globalización de la educación que se produce mediante plataformas tecnológicas ha permitido explorar nuevos horizontes sobre las intenciones pedagógicas y didácticas con las que cuenta la educación tradicional. En este sentido, en el marco de la educación virtual, el impacto es diferente y los alcances superiores a los de la educación tradicional. En este artículo se busca ampliar la visión de los nuevos retos de la didáctica aplicada en el campo de la educación virtual, digital o a distancia, en la que la participación de elementos tales como el autoaprendizaje, la disposición del tiempo, la ruptura de las barreras geográficas y la comprensión de la nueva relación docente-estudiante, entre otras, hacen parte del modelo formativo del estudiante virtual que escoge esta modalidad de estudio como una ruta hacia sus metas de formación profesional.
De esta forma, para la revista Virtu@Imente, en la sección «De cara a los expertos», se entrevistó a tres versados en educación virtual con el propósito de obtener sus puntos de vista sobre la virtualidad. El primero de los invitados es Héctor Alfonso Porras-Forero, docente-investigador del programa de Contaduría Pública y profesor catedrático de la Facultad de Estudios en Ambientes Virtuales del programa de Economía de la Universidad Ean; el segundo, el doctor Luis Fernando Pineda Ávila, médico cirujano con Especialización en Gerencia y Auditoría de la Calidad en Salud, cuenta con experiencia como gerente de prestación de servicios en diferentes hospitales de Bogotá y se desempeña como docente desde hace diez años en auditoría clínica en la especialización de la Universidad Cooperativa de Colombia. Por último, el tercero es Javier Isidro Rodríguez-López, coordinador virtual de la Facultad de Ciencias de la Salud de la Fundación Universitaria del Área Andina. 


\section{Panel de preguntas}

En la educación del siglo XX el docente era el centro del proceso de enseñanza-aprendizaje; hoy, en el siglo XXI, de acuerdo con los nuevos parámetros de la educación virtual, el estudiante es el mayor responsable de su proceso de aprendizaje (Ortega y Moreno, 2016). Con esto se establece que la disposición de la educación presenta un nuevo devenir y su metodología didáctica otro enfoque. La pregunta para los entrevistados es la siguiente: ¿Cuál sería el nuevo rol de la didáctica en el desarrollo y la implementación de nuevas estrategias de estudio enfocadas en esa optimización y en el enriquecimiento del aprendizaje?

\section{Héctor Porras}

La educación ha evolucionado a nuevos escenarios en los que las nuevas metodologías de aprendizaje, en todos los niveles de formación, se basan en tecnologías de la información, apoyadas con medios sincrónicos y asincrónicos en los cuales no es indispensable la presencia física de los participantes en el proceso de formación académica. Adicional a esto, la utilización de las nuevas plataformas educativas facilita las experiencias del conocimiento formativo y busca desarrollar mayores espacios de investigación y comunicación intercultural en los distintos escenarios en los que las tecnologías de la información y la comunicación o TIC muestran un horizonte por explorar.

\section{Luis Fernando Pineda}

Desde mi punto de vista, al tener en cuenta cómo la globalización que ha llevado al ser humano a una carrera contra el tiempo parece pasar cada vez más rápido, se ha hecho imperativo para la academia modificar las formas y responsabilidades tanto de los docentes como de los estudiantes en el aprendizaje y la generación de conocimiento. Este proceso de modernización propuso cambios en los que el docente ha cambiado la forma magistral y casi dictatorial de ofrecer las clases, de modo que las convierte en escenarios mucho más participativos y didácticos, es decir, el estudiante se incorporó de una manera más activa al desarrollo de la clase en un ambiente de trabajo organizado, orientado y controlado por el docente.

Desde la perspectiva del estudiante, también se ha cambiado la forma pasiva de recibir el conocimiento, pues con su participación se ha motivado a expresar diferentes formas de pensamiento, a desarrollar ideas innovadoras y a concebir nuevas propuestas de educación que los docentes deben involucrar en sus diferentes formas de enseñanza. 


\section{$\ldots \ldots \ldots \ldots \ldots \ldots \ldots \ldots \ldots \ldots$}

\section{Javier Rodríguez}

La evolución se ha dado en la medida en que las plataformas de learning management system o LMS se han ido adaptando a las necesidades de comunicación, interacción y consumo de contenido por parte de los diversos usuarios. El avance a modalidades como el m-learning o mobile learning genera muchas más exigencias para que la didáctica sea mucho más ágil y dinámica, de manera que responda a la evolución de cuarta generación de «darse la enseñanza en cualquier momento o en cualquier lugar adaptado a las generaciones actuales».

La estructuración académica y pedagógica de la educación virtual influye en la forma en que se llevará a cabo el aprendizaje tanto para el tutor como para el estudiante. Si la educación virtual tiene un orden en su estructuración, acorde con su modelo, va a permitir que se generen estrategias de aprendizaje, recursos de apoyo y actividades evaluativas que impacten de forma positiva en el proceso académico del estudiante.

Dentro del marco de transformación educativa que se ha logrado en los últimos años a nivel multicultural entre los países tanto de la región como externos, la aplicación de nuevas metodologías de estudio que buscan optimizar y enriquecer las herramientas de aprendizaje llevan a las instituciones educativas a replantear sus modelos de oferta académica con mayores elementos de análisis y vivenciales como resultado de la formación de los docentes. Entonces, ¿cuál sería el nuevo rol de la didáctica en el desarrollo e implementación de nuevas estrategias de estudio enfocadas en dicha optimización?

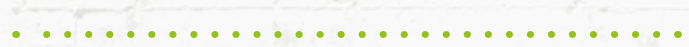

\section{Héctor Porras}

Desde hace 11 años soy docente de la Facultad de Estudios en Ambientes Virtuales o FEAV de la Universidad Ean, y la experiencia docente me ha permitido evidenciar la necesidad continua de cambios didácticos apoyados en las herramientas de las TIC. Aunque la metodología de la Ean tiene características de semipresencial, el diseño de nuevas ideas para la enseñanza, como docente, son un reto constante a fin de llegar con éxito a la puerta de las emergentes generaciones de estudiantes que ven en las aplicaciones ${ }^{1}$ y las plataformas digitales el nuevo mundo.

Otras alternativas que se prestan en la Ean son asesorías o clases a estudiantes que desarrollan actividades home school ${ }^{2}$, pero requieren asesorías en todos sus procesos, por tanto, cuentan con acompañamiento virtual para estar en capacidad de profundizar en aspectos específicos propios de cada bloque de estudio. 


\section{Luis Fernando Pineda}

Definitivamente, el cambio de mentalidad en los docentes es el aspecto más relevante. El tutor debe tener la capacidad de orientar las clases, desarrollar nuevas estrategias pedagógicas, manejar actividades de grupos y entender que la docencia es un asunto que implica el uso de nuevas tecnologías que harán parte de la modernización de la educación. De esta forma, el docente debe tener la capacidad de generar la responsabilidad que le corresponde al estudiante como actor fundamental en el marco de la relación docente-estudiante, a fin de alcanzar el éxito en el proceso formativo.

Sin embargo, dentro de la innovación educativa es aún parte fundamental el compromiso de estudiantes y docentes, sin el cual se puede ver afectada la calidad. La falta de responsabilidad de algunos estudiantes y docentes puede generar un impacto negativo en la generación de conocimiento futuro.

\section{Javier Rodríguez}

Si se tiene en cuenta que el acceso a internet es cada vez mayor a nivel global se puede inferir que, de la misma forma, la utilización de dispositivos móviles e inteligentes facilitará la educación virtual ulterior. Esta situación permite que las estrategias dirigidas a garantizar el aprendizaje y el desarrollo de las competencias sean mucho más dinámicas y totalmente adaptables a cualquier contexto social y cultural, situación que puede resultar en una aprehensión más ágil y fructífera del conocimiento. 
Figura 1. Generaciones de las modalidades educativas

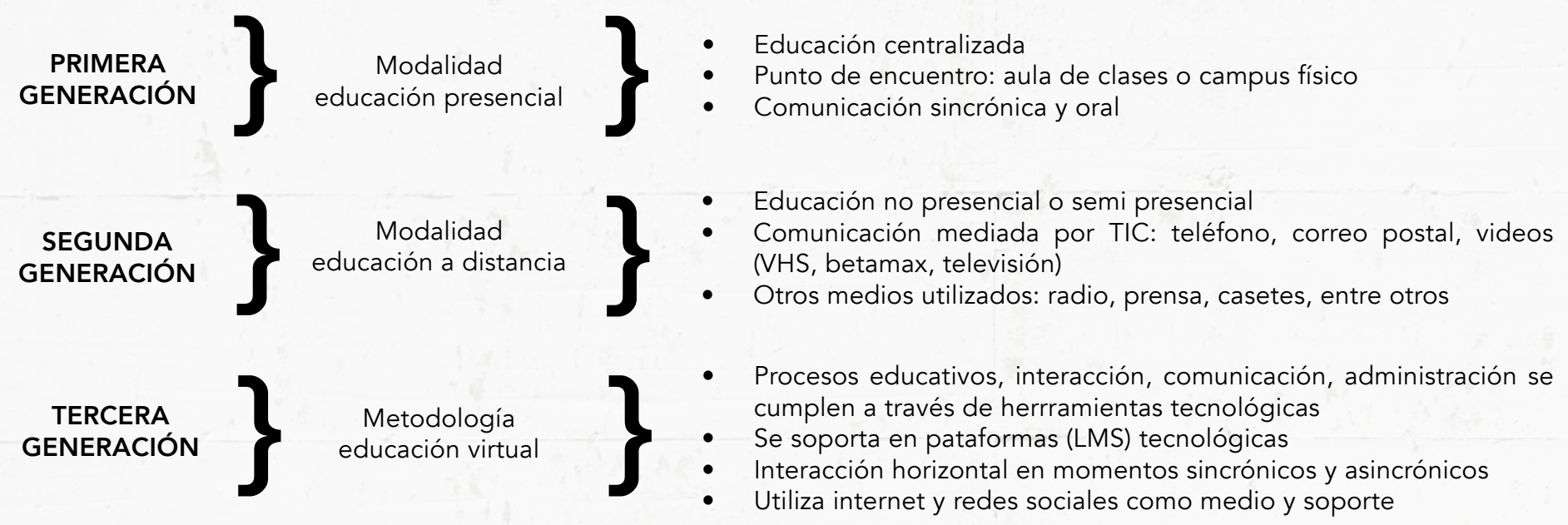

Fuente. Fundación Católica del Norte, 2005.

En la psicología del aprendizaje se plantea que el nuevo conocimiento interacciona con el conocimiento previo (Moreira, 2000, p. 8), por lo cual la modalidad de estudio virtual con respecto a la modalidad tradicional ha centrado sus esfuerzos en la interacción de varios grupos de interés que, de forma sinérgica, coactúan en la estructura de la educación virtual y a distancia. No obstante, la otra cara de la moneda ventila aspectos de gran relevancia en la educación formal y las insuficiencias de las que adolece. En este sentido, ¿cómo lograr la ampliación del radio de alcance de la educación virtual en las nuevas generaciones de tal forma que se sientan motivadas a adquirir el conocimiento de una forma distinta para hacer parte de una nueva sociedad?

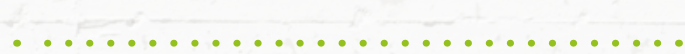

\section{Héctor Porras}

La presentación de las temáticas de estudio de la Universidad Ean establecen parámetros didácticos y pedagógicos encaminados a que los estudiantes realicen procesos de forma autónoma y tengan la libertad de escoger sus propias fuentes de información, así como de dar profundidad a las temáticas de su preferencia. La función del asesor docente es dar soporte, hacer revisión, direccionar y fortalecer los temas aprendidos; esta autonomía coadyuva al estudiante a sensibilizar la apropiación del rol formativo. 
Entre los parámetros a nombrar se establecen guías o talleres en bloques de estudio en los que se diseñan las temáticas a desarrollar, los objetivos y las estrategias contempladas para alcanzar el objetivo académico y el desarrollo de las habilidades. Se plantean objetos virtuales de aprendizaje u OVA, así como se establecen lecturas, ejercicios y tareas para el trabajo autónomo del estudiante dirigidos a fortalecer su proceso educativo. Adicional a esto, durante el ciclo académico se desarrollan tutorías mediante plataformas virtuales de aprendizaje en las que el estudiante interactúa con el docente. Por último, al final del periodo académico se programa un encuentro presencial que tiene una duración de ocho horas para cerrar el curso.

\section{Luis Fernando Pineda}

Los cambios deben darse, pero no solo en la metodología de docencia. Si bien los docentes se deben adaptar a los constantes cambios generacionales en la educación, no es menos cierto que existe falta de compromiso y responsabilidad por parte de los estudiantes frente a las nuevas tecnologías que son un tanto más flexibles. De cualquier manera, la modernización puede llevar a que se gradúen profesionales que no puedan responder a las iniciativas del país, debido a la falta de habilidades y competencias necesarias para comprender de una mejor manera el entorno.

\section{...........................}

\section{Javier Rodríguez}

La forma que accedan es a través de nuevas ofertas, flexibles, rápidas y convalidables por las empresas como parte de su formación para la labor corporativa. Generar alfabetización en el uso adecuado y aprovechable de la formación, a través del mobile learning, es uno de los retos del futuro, ya que las nuevas generaciones son dependientes de los dispositivos inteligentes $\mathrm{y}$, justamente, allí es a donde la oferta de educación moderna debe llegar.

La tendencia de las nuevas organizaciones empresariales es a buscar una mayor presencia de mercado, no simplemente local sino en el ámbito mundial. Una de las formas más económicas y rentables para tal objetivo se encuentra en la relación costo-beneficio de las páginas web. Aunado a esto, las grandes organizaciones buscan capacitar a su capital humano por medio de las plataformas virtuales, y es ahí donde la educación virtual cobra protagonismo, transformando la educación y abriendo puertas al aprendizaje individual y organizacional. Hoy en día, la capacitación virtual ocupa un lugar cada vez más destacado dentro de las organizaciones empresariales y educativas. De tal manera, el término electronic learning reúne las diferentes tecnologías, así como los aspectos pedagógicos de la enseñanza y el aprendizaje (Porras-Forero, 2013, p. 22). ¿Debería ser entonces diferente la didáctica establecida para programas de formación empresarial a la instituida en la formación académica del claustro universitario?, ¿por qué? 
$\ldots \ldots \ldots \ldots \ldots \ldots \ldots \ldots \ldots$

\section{Héctor Porras}

La metodología blended learning o b-learning, o mezcla entre la virtualidad y presencialidad, fortalece el trabajo autónomo del estudiante, ya que, a diferencia de la presencialidad, no se tiene un profesor que «dicta clases», sino que se establece un claro rol de tutor-aprendiz, quien acude a los encuentros con labores previas y consciente de la importancia de ser un juez de su propio desarrollo. El campo laboral difiere en algunas situaciones de lo que se presenta en la cátedra, por lo cual la Universidad Ean cuenta con un amplio grupo de docentes que han tenido experiencias empresariales de gran trayectoria y comparten su experticia laboral en la academia a fin de enriquecer el proceso formativo con la creación de modelos educativos más cercanos a la realidad empresarial como pilar del emprendimiento.

\section{Luis Fernando Pineda}

Para la actual generación de docentes ha sido más fácil involucrar la tecnología en la didáctica, con relación a lo que les compete a las generaciones anteriores, pues hoy se dispone de mayor acceso a la tecnología más avanzada, existe una comunicación más asertiva e incluso más facilidad para

${ }^{3}$ Esto es, de enseñanza, capacitación y aprendizaje online. consultar de forma ilimitada el conocimiento en cualquier biblioteca del mundo; todo esto difiere sustancialmente de las situaciones reales de hace 20 o 30 años.

Con relación a la tecnología, el beneficio está dado para adaptar a los docentes a la modernidad de la educación virtual y también a los procesos industriales-comerciales de los Estados, pues el desarrollo económico se encuentra directamente ligado a tecnología de punta.

Respecto a los resultados, es claro que el beneficio del uso de las tecnologías y las plataformas virtuales se ve reflejado en un cambio en la mentalidad de los directivos de las empresas que, a su vez, utilizan o construyen plataformas virtuales para sus procesos de inducción, reinducción y capacitaciones masivas, sin perder de vista que al involucrar sistemas de electronic learning o e-Learning ${ }^{3}$ deben aumentar la responsabilidad del estudiante-trabajador en el aprendizaje continuo y la generación del conocimiento.

$$
\ldots \ldots \ldots \ldots \ldots \ldots \ldots \ldots \ldots
$$

\section{Javier Rodríguez}

La respuesta es sí. Debe ser diferente, ya que en la empresa se enfoca a la labor y a fortalecer competencias que vienen débiles desde la formación universitaria. Además, la capacitación debe adaptarse a la cultura y a las dinámicas organizacionales. Sin embargo, lo que no debe cambiar es la forma de acceso: rápida, fácil y flexible. 
Adicionalmente, se deben generar reconocimientos en la formación empresarial, ya que muchos profesionales al permanecer tres o más años se vuelven maestros en la labor. Por tanto, esto debería convalidarse, además de compensarse con mejores condiciones laborales y económicas, de tal forma que auspicie el crecimiento personal y profesional de los empleados de las organizaciones.

\section{Conclusiones}

Es evidente el impacto positivo de las TIC en la educación, pues han permitido que se exploren distintos canales para el acceso al conocimiento. De esta forma, se entiende que el escenario virtual y digital es el nuevo camino para el aprendizaje de las generaciones futuras que entienden las distintas opciones digitales que ofrece la nube —cloud computing - como la ruta para sustituir el proceso formativo tradicional y cambiarlo por un modelo más dinámico e innovador que sea capaz de adaptarse a las necesidades de las nuevas generaciones.

La educación virtual se puede observar como un ser cambiante que se adapta a distintos escenarios —educativos, laborales, económicos, geográficos, temporales, etc.- con el único fin de transmitir conocimientos que solían adquirirse solo en atmósferas presenciales. En este sentido, permite asociarse a estructuras de costos más bajas para las instituciones académicas y las empresas, en las que su cobertura puede ser más amplia y darse en menor tiempo, sin condiciones geográficas y optimizando recursos humanos y financieros.
En el escenario micro se concluye que, en Colombia, se han logrado avances significativos en comunicación e infraestructura. No obstante, es necesario abogar por iniciativas gubernamentales —nacionales y municipalesque involucren los sectores marginados frente a la accesibilidad a internet, de tal forma que la educación virtual o digital, así como otros medios de apoyo informáticos, se conviertan en la nueva herramienta de inclusión y acceso al conocimiento. Internet y las aplicaciones para dispositivos móviles o inteligentes son la tendencia de la generación moderna y se prevé que las generaciones venideras encontrarán nuevas formas de acceder a la formación desde distintos escenarios, por esto, es necesario insistir en la democratización de la educación a través de medios virtuales y digitales, de tal forma que permitan entender la importancia del conocimiento como la existencia misma del ser humano. 
En una perspectiva macro del tema, desde el ámbito mundial el obstáculo de las distancias se ha superado con un solo clic y se ha convertido en la llave para nuevas oportunidades académicas y laborales. El acceso a la educación formal en el globo es uno de los grandes beneficios de los medios virtuales y digitales. Los estudios que antes solo se podían cursar de forma presencial han virado de manera sorprendente desde las mejores instituciones educativas del mundo y ahora se ofrecen virtuales. La facilidad para cursar un pregrado o un posgrado - maestría o doctorado- sin necesidad de la presencialidad es uno de los grandes avances de estos tiempos, pues el ahorro en costos asociados, como, por ejemplo, vuelos, hospedaje y manutención, ha permitido que cada vez más personas accedan al conocimiento.

Por último, un componente relevante de la virtualidad está asociado a la sostenibilidad y a la ecología, esta última entendida como la relación entre los seres vivos y el medio en el que viven, pues contribuye a que la huella de carbono se mitigue de diferentes formas y se mejore la relación con el medio ambiente: evita el desplazamiento de las personas a un lugar común, como, por ejemplo, a la universidad o el trabajo - lo que significa menos combustible-, optimiza la utilización de servicios públicos en la instituciones o corporaciones - lo que se traduce en menos gasto de agua y energía-, y disminuye los costos asociados de transporte, entre otros.

\section{Referencias}

Fundación Católica del Norte. (Comp.). (2005). Educación virtual. Reflexiones y experiencias. Medellín: Fundación Universitaria Católica del Norte. Recuperado de https://bit.ly/2NvM3cg

Moreira, M. A. (2000). Aprendizaje significativo: teoría y práctica. Ponencia presentada en el III Encuentro Internacional sobre Aprendizaje Significativo. Lisboa, Portugal, 11-15 de septiembre. Recuperado de https://bit.ly/2Z8YfVU

Ortega, S. C.; Moreno, C. (2013). La flexibilidad didáctica en entornos virtuales de aprendizaje. Revista Virtu@Imente, 1(2). Recuperado de https://bit.ly/2VgXSYk

Porras-Forero, H. (2013). La incorporación de las TICS (tecnologías de la información y las comunicaciones) como apoyo a la metodología presencial de educación superior en Colombia. Revista Economicus Heterodoxus. 2(1), 17-25. Recuperado de https://bit.ly/2BacyS5 